\title{
ÉTUDE DE LA TUTELLE COLLECTIVE SUR LES DROITS SOLIDAIRES
}

\section{ARTICLE ORIGINAL}

RIBEIRO, Danilo Miranda ${ }^{1}$

RIBEIRO, Danilo Miranda. Étude de la tutelle collective sur les droits solidaires. Revista Científica Multidisciplinar Núcleo do Conhecimento. Année. 06, Ed. 12, vol. 02, p. 152 à 167. Décembre 2021. ISSN : 2448-0959, Lien d'accès: https://www.nucleodoconhecimento.com.br/droit/droits-solidaires, DOI: 10.32749/nucleodoconhecimento.com.br/droit/droits-solidaires

\section{RÉSUMÉ}

Ce travail vise à construire une analyse juridique sur les éléments du processus collectif pour la protection des droits solidaires dans le système juridique brésilien. Parce que ses dispositions dans les lois sont espacées, l'étude de la protection collective est le problème d'un système étanche à l'air qui est difficile à comprendre. Dans le but de faire place à l'étude du processus collectif et d'écarter ce problème, cette recherche a pour problématique la discussion sur les principaux éléments du processus collectif, visant à démontrer qu'il s'agit d'un système procédural cohérent et harmonieux. La méthodologie utilisée sera la revue bibliographique. Ainsi, le travail présente en conséquence la systématisation des éléments fondamentaux de la compréhension de la protection collective, lorsqu'il s'agit d'aborder les dispositions juridiques et doctrinales sur la protection des droits solidaires, afin d'ouvrir la voie à une compréhension de la protection collective.

Mots-clés: Processus collectif, Droits solidaires, Procédure civile, Principes.

\footnotetext{
${ }^{1}$ Master en droits de l'homme et démocratie de I'UFPR-Université fédérale du Paraná. Baccalauréat en droit de I'UCSal-Université catholique de Salvador. ORCID : https://orcid.org/0000-0001-6503-9625.
}

RC: 103911

Disponible en: https://www.nucleodoconhecimento.com.br/droit/droits-solidaires 


\section{INTRODUCTION}

La protection collective est un instrument procédural essentiel pour la réalisation de la justice sociale en protégeant les biens juridiques qui n'appartiennent pas seulement à un individu, mais concernent la collectivité. II s'avère que les dispositions légales relatives à l'institut se situent dans des législations variées et ne dispensent pas de la compréhension constitutionnelle de la question, ce qui peut constituer un obstacle à l'enquête cohérente du processus collectif. Face à ce problème, cette recherche vise à offrir une réflexion sur les éléments essentiels de la protection collective des droits solidaires, tels que le concept de droits solidaires, la légitimité et la compétence. Ainsi, le processus collectif sera présenté de manière cohérente et harmonieuse avec les principes constitutionnels directeurs, afin d'éliminer l'hermétisme qui peut être perçu en connaissant la protection collective. La méthodologie utilisée sera la revue bibliographique des auteurs qui ont apporté une contribution importante à la compréhension de la protection des droits solidaires.

Du point de vue constitutionnel, le principe d'une procédure régulière (art. 5, LIV, $\mathrm{CF} / 88$ ) doit être interprété dans sa dimension collective, car il vise à réduire les formalités afin d'offrir un processus méta-individuel efficace et équitable. à la juridiction. Le système procédural collectif attribue une légitimité à différentes entités (ope legis), puisque la pertinence des intérêts protégés évoque la responsabilité des différents acteurs d'assurer les droits d'une collectivité. En ce sens, l'art. 103, du CDC place l'effectivité erga omnes ou ultra partes des décisions rendues dans le processus collectif (BELLINETTI ; SOUZA, 2019).

En ce qui concerne la classification, Zavascki (2005, p. 18) divise les intérêts collectivement en deux catégories. Le premier serait des intérêts individuels homogènes, qui auraient leur caractère collectif pour une question empirique, de contingence, non nécessaire, dans laquelle il y aurait plusieurs sujets, mais l'objet est divisible. La deuxième catégorie couvrirait les intérêts diffus et collectifs, constitués par ceux qui sont collectifs par leur essence, qui concernent un objet indivisible et un nombre incertain de sujets. En ce qui concerne les intérêts solidaire, 
il est possible de les concevoir dans deux domaines distincts: les droits individuels homogènes et les droits collectifs stricto sensu et diffus.

Selon Ronaldo Santos (2014), l'idée de protéger le droit collectif est d'assurer la justice sociale pour les groupes sociaux offensés ou de légaliser des situations qui n'avaient pas de protection judiciaire auparavant. Pour l'auteur, l'action collective avait pour antécédent le Bill of Peace, qui remonte à l'histoire du XVIle siècle :

[...] tratava de uma autorização para o processamento de uma ação individual sob a forma coletiva, concedida nas hipóteses em que o autor requeria que o provimento da demanda englobasse os direitos de todos os indivíduos que estivessem em igual condição de litígio, para que a questão fosse tratada de modo uniforme e com vistas a evitar a multiplicação de processos (SANTOS, 2014, p. 238).

En mettant l'accent sur la relation entre les droits solidaires et les droits fondamentaux, Hachem comprend que les premiers portent les mêmes éléments qui seraient liés aux droits fondamentaux des trois générations, et pas seulement à la troisième, deuxième ou première génération:

Por conta da complexidade de sua natureza jurídica e estrutura
normativa, todos os direitos fundamentais reúnem
concomitantemente a totalidade dos traços que supostamente seriam
peculiares a cada uma das gerações: (i) impõem deveres negativos
ao Estado; (ii) dirigem ao Poder Público obrigações de fornecer
prestações fáticas e normativas; (iii) ostentam a titularidade
transindividual alegadamente exclusiva dos "direitos de terceira
geração", bem como, simultaneamente, a titularidade individual
pretensamente típica dos "direitos de primeira e segunda geração"
(HACHEM, 2013, p. 621).

Grâce aux revendications collectives, l'accès à la justice d'une collectivité indéterminée ou d'un groupe déterminé de personnes est amplifié. La socialisation et la démocratisation du processus sont soulignées comme des avantages de ces actions, avec la réalisation des droits et l'altération de la réalité sociale; la possibilité de corriger un illicite qui ne serait pas postulé dans une demande individuelle avant sa petite valeur particulière, mais qui a collectivement un grand impact social; l'égalité entre les plaideurs; la molecularisation de la demande et la réalisation du principe de sécurité juridique et de confiance légitime face à des décisions uniformes 
pour des affaires similaires; la réduction des demandes dans le système judiciaire, entre autres.

Plus précisément, les intérêts individuels homogènes portent le vêtement des intérêts individuels, mais face à certaines configurations de la société, il est nécessaire d'être protégé collectivement. La nomenclature elle-même traduit déjà une partie du concept de cet intérêt, selon la leçon de Zavascki:

$\mathrm{Na}$ verdade, a própria denominação interesses individuais homogêneos já exibe dois enunciados: de um lado, trata-se de interesses ontologicamente individuais, não coletivos; de outro lado, que consistem numa multiplicidade de interesses individuais apresentando um determinado grau de uniformidade ou homogeneidade, sua característica mais importante (ZAVASCKI, 2005, p. 18).

Selon l'article 81 de la loi 8.078/91, les intérêts individuels homogènes sont fondés sur l'idée d'origine commune, sur le fait préjudiciable qui a affecté et violé d'innombrables personnes dans leur individualité, sans exigence d'une relation juridique fondamentale entre les titulaires ou entre les parties, ni d'une unité quant aux faits ou à la temporalité (ZAVASCKI, 2005, p. 21).

Les intérêts individuels homogènes sont ceux qui ont à l'origine un fait néfaste qui a affecté une collectivité et dépasse la sphère de l'intérêt individuel. Par conséquent, ces droits ont une origine commune, qui découle de la conduite omissive ou commissaire de la partie adverse qui affecte les droits qui échappent au simple aspect individuel et créent la prétention collective, apportant à la discussion des questions supérieures à la simple lide singulière.

Selon la leçon de Zavascki (2005, p. 21), ils sont divisibles, appropriés individuellement, disponibles, transférables et représentés devant le tribunal par le titulaire lui-même, épisodiquement transindividuels, subjectivement déterminés et directement réparables.

Toutefois, la caractéristique de l'individualité ne s'écarte pas du contenu solidaire éminent des droits individuels homogènes. Lorsqu'ils sont considérés collectivement, 
ces droits dépassent de loin la sphère d'intérêt particulier de leurs détenteurs. La caractéristique collective ne réside pas dans l'impossibilité d'être approprié individuellement ou dans le fait que leurs détenteurs soient indéterminables, mais dans la portée que révèle la violation de la société (ZAVASCKI, 2005, p. 21-22). L'atteinte à des intérêts individuels homogènes viole la paix sociale et favorise un grand malaise social, ce qui génère un effet synergique, puisque les deux sont renforcés.

II serait également possible de nommer des intérêts individuels pertinents ou des intérêts communs, ce qui n'a pas été fait parce que l'homogénéité est essentielle pour avoir la possibilité de protéger collectivement la loi (ZAVASCKI, 2005, p. 19).

Toujours en ce qui concerne les droits solidaires, les catégories de droits diffus et collectifs stricto sensu se posent. Premièrement, les droits diffus sont les intérêts collectifs qui sont indivisibles, dont les titulaires sont indéterminés et n'ont pas de relation juridique entre eux (DIDIER JUNIOR; ZANETI JUNIOR, 2014, p. 76-78). La notion de droits diffus est trouvée par l'analyse de l'article 81 , I, du Code de protection des consommateurs, qui sont conceptualisés comme des intérêts solidaires de nature indivisible, dont les titulaires sont indéterminés et liés par un fait commun.

Les droits diffus sont ceux qui appartiennent à une collectivité de sujets indéterminés, portant des droits indivisibles et liés par une situation de fait (superveniente). C'est-à-dire que la relation entre les titulaires est née à un moment postérieur au préjudice, puisqu'il n'y avait pas de lien entre le blessé à un moment antérieur et le fait qui justifiait une protection collective (DIDIER JÚNIOR; ZANETI JúNIOR, 2014, p. 78).

Ensuite, il y a les droits collectifs stricto sensu, qui trouvent une définition dans l'article 81, II de la CDC. Ce sont des droits solidaires, à la différence que leurs titulaires sont certains groupes de personnes définis, c'est-à-dire qu'il existe une relation juridique de base entre les titulaires, qui sont indéterminés mais déterminables. C'est-à-dire que la différence entre les droits diffus et les collectifs 
stricto sensu est que les premiers ont une propriété indéterminée et les seconds ont certaines catégories collectives en tant que détenteurs, de sorte que les personnes peuvent être déterminées (DIDIER JÚNIOR; ZANETI JÚNIOR, 2014, p. 78-79).

Pour les intérêts collectifs stricto sensu, il est essentiel d'identifier quel groupe d'individus a été lésé, car le droit est indivisible et les titulaires sont ceux qui appartiennent à une certaine catégorie (DIDIER JÚNIOR; ZANETI JÚNIOR, 2014, p. 78).

Il convient de noter que la relation juridique de base doit être antérieure à l'événement dommageable. C'est-à-dire que la relation juridique entre les titulaires ne provient pas du dommage, comme cela se produit dans les droits diffus, mais de l'adhésion à la catégorie de travail, à l'association, à certaines sociétés et à d'autres causes qui ont précédé le préjudice (DIDIER JÚNIOR; ZANETI JÚNIOR, 2014, p. 78). De cette manière, les titulaires du droit sont entièrement déterminables et appartiennent à une collectivité individualisable.

\section{LÉGITIMITÉ}

Dans les situations de juridiction singulière, presque toujours, les sujets en question n'intéressent que les parties impliquées dans le cours, sans avoir de poids direct sur les personnes qui ne sont pas impliquées dans le pôle passif ou actif du cours.

Traditionnellement, la légitimité active de la protection des intérêts individuels exige un examen de la propriété et remonte à l'héritage du droit romain (DIDIER JÚNIOR; ZANETI JÚNIOR, 2014, p. 79). D'autre part, l'institut de droit au nom d'une autre personne n'est pas quelque chose de beaucoup plus actuel, puisque « [...] au Moyen Âge, il n'y avait pas encore le concept de personnalité juridique, de sorte qu'il n'était même pas possible de considérer que quelqu'un était représenté par une autre personne ; la légitimité ordinaire était la règle absolue » (ZAVASCKI, 2005, p. 11).

Dans l'ordre juridique brésilien, la règle générale est prévue à l'article 18 du Code de procédure civile, qui place la propriété du droit comme une exigence fondamentale 
pour sa discussion, puisque la discussion du droit qui appartient à la personne d'autrui appartient, sous réserve des cas expressément prévus par la loi, demeure.

Cependant, en plus de la légitimité ordinaire, représentée par l'article 18 du CPC, il existe, à titre exceptionnel, une légitimité active extraordinaire, qui est admise dans les cas expressément prévus par la loi, dans lesquels quelqu'un plaidera le droit au nom du titulaire. Dans ce cas, il existe une option législative dans les cas où il sera possible d'invoquer le droit d'autrui devant les tribunaux.

Outre les cas prévus par l'organe juridique, les hypothèses de légitimité extraordinaire doivent être considérées comme une conséquence logique du système juridique. Neves (2016) est l'un des auteurs qui commentent la possibilité d'une légitimité extraordinaire en l'absence d'autorisation légale expresse :

Apesar de o art. 18, caput, do Novo CPC prever que a legitimação depende de autorização expressa da lei, a melhor doutrina entende que, além da previsão legal, também se admite a legitimação extraordinária quando decorrer logicamente do sistema, como acontece com a legitimação recursal do advogado em apelar do capítulo da sentença que versa sobre seus honorários advocatícios (NEVES, 2016, p. 188).

La flexibilité par rapport à la règle de l'article 18 du CPC apparaît comme un meilleur moule à l'efficacité de l'institut, propre à défendre les intérêts du collectif. Cependant, il est averti: la flexibilisation n'apporte pas un suivi aussi inévitable une procédure plus simple.

II est à noter que le même droit peut être garanti à des personnes de différents groupes sociaux, ce qui se produit avec une certaine fréquence, par exemple en cas de violation des garanties sociales. Par conséquent, il est difficile d'affirmer qu'il existe une légitimité pour représenter tous ceux qui sont intéressés, compte tenu de la grande différence culturelle, sociale ou économique qui existe entre les titulaires de droits dans les revendications collectives.

C'est dans ce contexte (ZAVASCKI, 2005, p. 15) que les difficultés de défense des intérêts collectifs ont commencé. Si les individus sont différents, comment supposez- 
vous que l'un d'eux a la légitimité d'exiger pour tout le monde la défense de cet intérêt commun? Comment étendre les effets de la protection collective à ceux qui ne sont pas devant les tribunaux si l'on ne peut présumer de l'intérêt légitime de ces autres? Comment exigez-vous que de grands groupes comparaissent devant les tribunaux? Comment faire cela si les membres du groupe ne sont même pas déterminés?

Compte tenu de l'indétermination des titulaires, d'une grande différence qui existe entre eux et de la difficulté pratique de tous à porter certaines questions devant les tribunaux, la légitimation des entités qui ont pour but la recherche et la défense des intérêts d'une collectivité.

De cette façon, les collectifs légitimés ne sont pas titulaires des droits qui sont légitimés pour plaider et les titulaires de ces droits ont la propriété active vidée (NEVES, 2016, p. 189). Par excellence, dans la lettre de Zavascki (2005, p. 15), la légitimité active dans les revendications collectives pourrait être citée comme la représentation de l'absent.

Dans la juridiction collective, il n'y a pas de lien direct entre la propriété du droit et la légitimité, mais il existe une situation juridique qui amène la capacité de certaines institutions à défendre des intérêts qui ne se limitent pas à l'idée d'individualisme, ce qui amène la configuration d'une légitimité extraordinaire. En ce sens :

A legitimação ao processo coletivo é extraordinária: autoriza-se um
ente a defender, em juízo, situação jurídica de que é titular um grupo
ou uma coletividade. Nãa há coincidência entre o legitimado e o
titular da situação jurídica discutida (DIDIER JUNIOR; ZANETI
JUNIOR, 2014, p. 21). Le système juridique brésilien a accepté la possibilité d'assumer la capacité de certaines institutions à être légitimées pour défendre des revendications collectives (ZAVASCKI, 2005, p. 102). C'est-à-dire que ceux légitimés aux fins d'actions collectives découlent de l'établissement légal, comme le prévoit l'article 5 de la loi sur l'action civile publique, qui énumère le ministère public, le bureau du défenseur public, I'Union fédérale, les États, le district fédéral, les municipalités et d'autres 
sujets, en tant que représentants du collectif et en mesure d'intenter une action civile publique.

Dans le même mouvement du LACP, l'article 82 du Code de protection des consommateurs souligne la légitimité active du ministère public, de l'Union, des États et municipalités, des municipalités, des entreprises publiques, des fondations, des sociétés ou associations d'économie mixte pour défendre les droits homogènes diffus, collectifs ou individuels des consommateurs.

En outre, en ce qui concerne la légitimité active aux fins des actions collectives, Zavascki (2005, p. 132) soulève un aspect controversé qui entoure la possibilité pour le ministère public de défendre des intérêts individuels homogènes, puisque le CRFB/88 ne confère pas cette fonction au parquet.

La langue vernaculaire constitutionnelle, dans son article 129, point III, n'énumère pas la défense d'intérêts individuels homogènes comme l'une des attributions du ministère public, dont les activités sont liées au texte constitutionnel. Ainsi, il est possible d'interpréter comme une clôture juridique l'absence de détermination à défendre ces intérêts (ZAVASCKI, 2005, p. 132).

D'autres plaident pour la légitimité illimitée du ministère public (ZAVASCKI, 2005, p. 133). Selon l'article 129, point IX, du CRFB/88, il appartient au député d'exercer des fonctions compatibles avec son objet, ce qui inclut la défense des intérêts sociaux et individuels indisponibles, à la lumière de l'article $127 \mathrm{du}$ CRFB/88. Pour ce courant, les intérêts solidaires ont une présomption de pertinence sociale, ce qui permet au parquet d'agir (ZAVASCKI, 2005, p. 133).

Enfin, il y a la thèse de la légitimité restreinte, qui émerge comme une conséquence de l'idée que le ministère public aurait une légitimité illimitée, avec l'avertissement que les questions d'intérêt social ne pourraient être que celles qui contiennent des congruences avec les fonctions constitutionnelles du parquet (ZAVASCKI, 2005, p. 132), ceci sur la base de l'article 127, CRFB/88, sans l'effort de présumer de la pertinence pour pouvoir apporter une légitimité au procureur de la République. 
En ce qui concerne la légitimité du ministère public dans la protection d'intérêts individuels homogènes, Zavascki conclut que la réponse à cette question réside dans l'analyse des costumes juridiques, car c'est dans la loi que réside la notion d'intérêt de pertinence sociale, qui assurera la légitimité active du parquet (ZAVASCKI, 2005, p. 132). De cette façon, Zavascki enseigne que les intérêts de la pertinence sociale sont constitués par: «l'imposition de l'indisponibilité, l'attribution directe de la légitimité au ministère public pour sa défense et la caractérisation expresse de la pertinence sociale » (ZAVASCKI, 2005, p. 136).

La délinquance n'est pas controversée. En l'absence de l'aspect de la pertinence sociale dépeint par Zavascki, il n'est pas nécessaire de parler de la légitimité du parquet pour agir en défense d'intérêts individuels homogènes.

Enfin, il convient de souligner les caractéristiques que Didier révèle dans la légitimation collective active, en tant que règlement par l'article 5 de la loi fédérale $n^{\circ}$ 7,347/85 et l'art. 82 de la CDC, étant portés par des entités qui n'agissent pas en leur propre nom, puisque les titulaires n'ont pas de capacité postulatoire:

Enfim, o certo é que a legitimação coletiva possui as seguintes características: a) está regulada, inicialmente, por lei (art. 5ำ da Lei Federal no 7.347/85; art. 82 do CDC etc.); b) é conferida a entes públicos, privados e despersonalizados, e, até, ao cidadão, na ação popular; c) o legitimado coletivo atua em nome próprio na defesa de direitos que pertencem a um agrupamento humano (pessoas indeterminadas, comunidade, coletividade ou g rupo de pessoas, titulares de direitos individuais abstratamente considerados, na forma do a1i. 81 do CDC e seus incisos); d) esse agrupamento humano não tem personalidade judiciária, portanto não pode atuar em juízo para proteger os seus direitos, 26 cuja defesa cabe aos legitimados coletivos, que possuem legitimação autônoma, exclusiva e concorrente e simples (DIDIER JUNIOR; ZANETI JUNIOR, 2014, p. 212).

\section{COMPÉTENCE}

La compétence est un élément fondamental pour la satisfaction d'une procédure régulière. La défense des droits solidaires se fait en faveur d'une collectivité, qui peut 
être séparée et sans aucun lien qui l'unit, ce qui attire l'attention sur le moment de la fixation de la compétence.

Pour la fixation de la compétence territoriale dans les revendications collectives, Neves énumère sept étapes nécessaires à la divulgation du jugement compétent. II est souligné que la fixation de la compétence territoriale est une conséquence de l'analyse de la législation procédurale, de la même manière qu'elle se fait dans les cas de protection singulière, puisqu'il s'agit d'une matière liée à la loi traitée de manière isonomique à la protection individuelle (NEVES, 2016, p. 168).

Toutefois, il est nécessaire de souligner que la législation régissant la protection des droits collectifs peut établir la compétence émanant de certains tribunaux ou organismes atypiques, à la manière de ce qui peut se produire dans le cadre du processus collectif spécial, de l'action populaire, de l'action civile publique, de l'injonction collective, du mandat collectif et de l'action pour faute administrative.

En raison de la pertinence des mesures, il convient de noter que la compétence dans la sphère collective est réglementée par les arts. 2, la loi sur l'action civile publique et 93 du code de la protection du consommateur, qui établissent comme critère de compétence territoriale le lieu du dommage.

En outre, selon la législation du LACP, la compétence serait territoriale et fonctionnelle, ce qui soulève le débat sur la question de savoir si la compétence serait due aux forums, à la question territoriale ou à une plus grande efficacité et facilité, en vertu du critère fonctionnel. Néanmoins, il est certain que la compétence territoriale est absolue, ce qui conduit à son respect obligatoire.

Après avoir déterminé le for compétent, la recherche du jugement compétent commence, qui se fera à partir des lois d'organisation du pouvoir judiciaire et du CPC. En effet, la compétence du jugement est affectée par la compétence territoriale, et non l'inverse (NEVES, 2016, p. 186). 


\section{EXÉCUTION DU JUGEMENT RENDU DANS LE CADRE DE RECOURS COLLECTIFS}

La base de l'exécution d'un jugement individuel est portée par l'exécution d'un jugement collectif, qui doit avoir lieu comme une continuation du processus de connaissance, après le moment où le débiteur n'a pas respecté le commandement de la peine. La phase de mise en œuvre sera respectée par l'exécution d'un jugement, selon les circonstances prévues à l'article 515 du CPC.

En ce qui concerne les droits diffus et collectifs stricto sensu, la protection du droit matériel se fera par l'exécution collective, qui se déroulera au profit de la communauté, qui se fera à travers les aspects utilisés dans l'exécution individuelle, sans aucune spécialité procédurale en raison de la nature collective de l'intérêt (NEVES, 2016, p. 392-393).

De l'exécution peut découler une injonction (interdiction de l'illégalité) ou réparation in natura, commeannulation des dommages-intérêts, ou en espèces, qui, dans ce cas, doit être renvoyée à l'entité juridique de droit public qui a subi le préjudice ou au Fonds des droits diffus (art. 13 de la loi fédérale $\left.n^{\circ} 7.347 / 1985\right)$ (DIDIER JÚNIOR; ZANETI JÚNIOR, 2014, p. 412).

En ce qui concerne la légitimité, toute partie intégrante du pôle actif peut favoriser l'exécution, même si elle n'a pas participé à la phase de cognition, selon l'interprétation de l'article 15 de la loi 7.347, qui énumère comme exigence le laps de temps de 60 jours du transit final afin que de nouveaux sujets participent au processus en vue d'initier la phase de mise en œuvre (DIDIER JÚNIOR; ZANETI JÚNIOR, 2014, p. 413). C'est-à-dire que l'exécution collective de droits diffus ou collectifs stricto sensu peut se faire par tout extraordinaire légitimé, en respectant le cours temporel du transit final. Ceci est également prévu à l'article 15 du CBPC-IBDP et à l'article 26 du CBPC-UERJ/UNESA. 
II est souligné l'obligation de proposer l'accomplissement du jugement comme une attribution essentielle au parquet, dans le souci des intérêts collectifs (NEVES, 2016, p. 391).

Toutefois, il convient de noter que l'obligation du procureur d'exécuter un jugement en matière civile publique naît après 60 jours du jugement définitif de la sentence, conformément à l'article 15 de la loi 7.347/85.

En ce qui concerne l'action populaire, l'obligation du ministère public d'exécuter la peine est pendante lorsque le citoyen est inerte pendant plus de 60 jours, à compter de la publication du jugement au deuxième degré, conformément à l'article 16 de la loi 4.717/65. C'est-à-dire qu'il y aurait l'obligation fonctionnelle du parquet mobile de se conformer à une peine provisoire, avant la possibilité de modification du commandement de la phrase.

Par conséquent, il existe également la possibilité d'une application individuelle dans l'exécution des droits diffus et collectifs stricto sensu, selon la leçon de Didier:

\footnotetext{
É também possível que a sentença proferida em um processo em que se discutem direitos difusos ou coletivos seja utilizada por um indivíduo corno título de uma execução individual, tendo em vista a extensão in utilibus da coisa julgada coletiva ao plano individual, já examinada. Obviamente, antes de executar a decisão, o indivíduo deverá proceder à liquidação do seu crédito, em que deverá demonstrar, inclusive, que é titular de um crédito (liquidação com thema decidendum mais amplo do que a liquidação individual prevista no CPC, restrita à decisão sobre o valor ou sobre a coisa a ser executada) (DIDIER JUNIOR; ZANETI JUNIOR, 2014, p. 415).
}

En ce qui concerne les droits individuels homogènes, qui sont des droits individuels agrégés par leur origine commune, l'exécution sera effectuée individuellement par les titulaires. C'est-à-dire que la forte composante de collectivité qui existait dans la phase de connaissance a ralenti dans la phase d'exécution, puisque l'intérêt individuel sera traité comme collectif dans la phase d'exécution (NEVES, 2016, p. 394). Quant aux forums, l'article 516 du CPC impose une règle stricte, un critère de compétence absolue, qui conduirait à l'exécution d'un jugement pour le jugement qui a tranché l'affaire au premier degré de compétence. 
L'interprétation de cette disposition juridique inclut l'exécution individuelle d'un jugement collectif, cependant, par des critères pratiques, Neves (2016, p. 376) défend comme compétent pour le règlement individuel du jugement collectif le for $\mathrm{du}$ domicile du liquidateur, de sorte qu'il n'y a pas de grande concentration des procédures dans le district qui a statué sur l'affaire au premier degré de compétence et moins de charge pour l'exequente.

Toutefois, il convient de noter qu'il y a eu un veto présidentiel en ce qui concerne le paragraphe unique de l'article 97 de la $C D C$, ce qui exclut l'hypothèse selon laquelle le for de l'exécution d'un jugement collectif est choisi comme celui du domicile de l'exequente.

Selon Zavascki (2005, p. 164), il n'y a aucun moyen de considérer le veto comme inutile, puisqu'il ouvre la voie à l'interprétation et révèle des raisons qui devraient être prises en considération, puisqu'elles sont examinées par les législateurs.

Compte tenu de la détermination expresse de la compétence pour l'exécution de la décision, c'est celle de la décision qui a jugé l'affaire au premier degré de compétence, selon l'article 516, II de la CPC, les doutes sur la compétence n'existent pas.

Enfin, il y a un point controversé à l'article 98 de la $C D C$, en ce qui concerne la possibilité pour le ministère public et les organismes publics (légitimés de l'article 82 de la $C D C$ ) de promouvoir l'exécution d'intérêts individuels homogènes, puisque la sphère collective est vidée au moment de l'exécution des intérêts individuels. Soulignant la leçon de Zavascki:

Quanto ao Ministério Público e órgãos públicos, não se vislumbra circunstância na qual tais entes teriam algum interesse em promover liquidação e execução específicas em favor de particulares, ainda mais mediante representação. Em consequência, é certo não possuir - Ministério Público legitimidade para promover 0 tratamento individualizado da sentença genérica, mas não porque ele não tem poderes para agir em nome próprio em favor das vítimas e sucessores e sim porque, nessa hipótese, não se trata de defesa de interesses sociais ou individuais indisponíveis (ZAVASCKI, 2005, p. 163).

RC: 103911

Disponible en: https://www.nucleodoconhecimento.com.br/droit/droits-solidaires 
Enfin, il est intéressant de noter que même ceux qui ne font pas partie du processus peuvent se plaindre de ce qui a été proposé dans un jugement collectif, puisque son but est de donner du concret aux droits de la communauté:

Diante disso, os instrumentos processuais utilizados para tutela dos interesses transindividuais devem ser adaptados à natureza do interesse substancial em questão, de modo a lhe proporcionar uma maior efetividade. Para tanto, algumas alterações legislativas foram feitas com a finalidade de atender esse escopo. Dentre elas menciona-se a tratativa dada à sentença coletiva, à coisa julgada e à execução de sentença coletiva. A sentença coletiva, dada sua natureza, possui um alcance diferenciado no que se refere aos indivíduos por ela albergados, podendo atingir, inclusive, aquele que não foi parte no processo, dada a extensão secundum eventum litis da coisa julgada coletiva ao plano individual (ALFREDO, BALEOTI, 2012, p. 435).

\section{GARDIENS DE L'URGENCE DANS LES ACTIONS COLLECTIVES}

D'une manière générale, la protection de l'urgence dans les actions collectives ne présente pas de divergence de raisonnement par rapport aux actions individuelles (DIDIER JÚNIOR; ZANETI JÚNIOR, 2014, p. 342), qui apporte l'incidence de la CPC lorsqu'il y a les exigences énoncées dans son article 300 .

Cependant, il existe certaines procédures spéciales qui prévoient cette technique procédurale, comme l'article 12 du LACP, qui permet d'accorder une protection précoce dans la défense de certains intérêts collectifs (DIDIER JÚNIOR; ZANETI JúNIOR, 2014, p. 342).

À titre d'exemple, l'article 4 du LACP prévoit également la possibilité de mesures injonctives ayant pour objet de prévenir les dommages aux biens juridiques qui y sont décrits. À juste titre, Didier conduit à la rectification des dispositions de cet article, dès lors que la mesure serait satisfaisante, puisqu'elle accorderait le droit recherché par la provocation du pouvoir de justice, et non une mesure conservatoire:

Embora mencione expressamente a tutela cautelar, a redação do dispositivo não dá margem a dúvida: não se trata de tutela cautelar, mas, sim, tutela inibitória, que é satisfativa e visa exatamente obter providência judicial que impeça a prática de ato ilícito e, por

RC: 103911

Disponible en: https://www.nucleodoconhecimento.com.br/droit/droits-solidaires 
consequência, a ocorrência de um dano (DIDIER JÚNIOR; ZANETI JÚNIOR, 2014, p. 343).

Il est important de souligner certains obstacles à l'octroi d'une protection provisoire dans les recours collectifs, tels que les dispositions de l'article 2 de la loi fédérale $n^{\circ}$ 8 437/1992 et du $§ 2$ de l'article 22 de la loi $n^{\circ}$ 12.016/2009, qui limitent l'octroi d'une injonction dans un mandat de sécurité collective ou une action civile publique à l'audition du représentant judiciaire de l'entité juridique de droit public, doit être effectuée dans un délai maximum de 72 heures.

En outre, il est similaire à ce qui se passe pour l'octroi d'une injonction dans le conflit collectif pour la possession de biens, qui est subordonné au respect des exigences énoncées à l'article 565 du Code de procédure civile.

\section{RECORRECTABILITÉ DANS LES ACTIONS COLLECTIVES}

II n'y a pas de support récursif spécifique à utiliser dans les recours collectifs. Ce qu'il y a d'exceptionnelles mentions de la récorrécabilité dans l'application dans les revendications collectives, qui apporte une application générale du système récursif du Code de procédure civile (NEVES, 2016, p. 324)

En ce qui concerne la contestation d'une décision d'interlocution, l'article 1015 de la CPC énumère la liste des hypothèses qui ouvrent la portée au préjudice de l'instrument. Selon l'autorisation expresse de cet article, dans son paragraphe XIII, d'autres cas prévus par la loi permettent la gestion de l'instrument lésé, dans lequel l'article $19, \S 1$, de la loi 4.717/65, avec la disposition qu'il convient d'aggraver l'instrument en raison d'une décision d'interlocution dans le cadre de l'action populaire.

Selon Neves (NEVES, 2016, p. 325), en raison du microsystème collectif, la norme susmentionnée devrait être applicable à toutes les autres actions collectives, ce qui permettrait l'interposition d'un instrument de préjudice dans toute décision interlocutoire découlant d'un processus collectif. 
En ce qui concerne la légitimité récursive, il est intéressant d'analyser qu'il existe des exceptions à la règle 996 du CPC, qui établit la partie qui succombe, les tiers intéressés et les procureurs comme des actifs légitimes aux appels.

Il convient de souligner que l'article 996 de la CPC traite de l'intérêt récursif et non de la légitimité récursive, puisque, lorsqu'il s'agit d'une partie qui succombe, une analyse de la succombation est faite, ce qui conduit à une analyse concrète de la décision qui remet en question l'intérêt récursif (NEVES, 2016, p. 330). La légitimité n'a rien à voir avec l'intérêt, car c'est une partie légitime de recourir à celui qui gagne ou perd, le critère d'établissement de la légitimité est totalement indépendant du contenu de la décision.

II convient de souligner un thème important: conformément à la règle de l'article 19, $\S 2$, de la loi $4.717 / 65$, tout citoyen peut faire appel des jugements et décisions rendus à la suite d'une action populaire. II s'avère que, selon la règle énoncée à l'article 996 du CPC, le citoyen qui ne compose pas le pôle actif du cours ne convient pas en tant que partie infructueuse ou tierce partie, puisque le droit de commenter dans l'action populaire est diffus, ce qui supprime la notion de tiers intéressé par le seul paragraphe de l'article 996, CPC. Ainsi, le citoyen ne compose pas la liste des sujets qui ont une légitimité récursive, mais trouve la permission dans le LAP de faire appel.

Quant aux effets récursifs (obstinents, translattifs, expansifs, substitutifs, régressifs et différés), il n'y a pas de particularité lorsqu'ils sont générés dans le processus collectif. Seulement en ce qui concerne l'effet suspensif, il est nécessaire d'examiner la législation concernant chaque action, afin de vérifier l'absence ou la présence de chaque effet récursif mentionné (NEVES, 2016, p. 332).

Il convient de souligner la législation régissant l'action civile publique, qui confère au juge un effet suspensif sur les recours ayant pour portée de supprimer un préjudice irréparable aux parties, en fonction de l'article 14 de la loi $7.347 / 1985$, qui a permis de comprendre que les recours formés dans le cadre de l'action civile publique n'ont pas d'effet suspensif uniquement en raison de leur simple interposition (NEVES, 
2016, p. 332). Ainsi, le recours, qui, en règle générale, a des effets suspensifs, en vertu de l'article 1012 du code de procédure civile, doit respecter les exigences énumérées à l'article 14 du LACP (éviter un préjudice irréparable mis à part) pour éviter que le jugement rendu dans l'action civile publique ne produise des effets immédiats.

\section{CONSIDÉRATIONS FINALES}

Compte tenu de la discussion menée par ces travaux, il est possible d'observer que le texte du CRFB/88 gagne en force et en efficacité avec la protection des droits collectifs, à la manière d'une recherche de la matérialisation des garanties sociales qui sont prévues dans la langue vernaculaire constitutionnelle et peuvent être réclamées judiciairement.

Un tel instrument est extrêmement intéressant pour que la justice sociale soit réalisée dans une société de masse, où la violation d'un droit peut aller au-delà de la sphère individuelle et atteindre une collectivité de personnes. C'est pourquoi, afin de répondre au problème présenté initialement, le parti pris constitutionnel de garantir les droits solidaires a été mis en évidence en conférant un caractère unique aux dispositions juridiques, qui visent à garantir des droits collectifs en confluence avec la réalisation du principe de justice sociale et de procédure régulière.

En explorant les éléments essentiels de la protection collective, un système harmonique a été présenté et qui trouve une cohérence pour assurer la protection du bien juridique de l'ensemble de la collectivité. Ainsi, le problème de l'hermétisme de ce système procédural a été surmonté, car il a été abordé de manière à discuter de sa matrice constitutionnelle et de ses fondements juridiques. Par conséquent, ce qui pourrait ressembler à un système déconnecté, parce qu'il est normalisé dans diverses législations, est interprété comme un instrument intégré qui vise à garantir des droits qui appartiennent non seulement à une personne, mais à la société. 


\section{RÉFÉRENCES}

ALFREDO, Luciana Romaneli Rodrigues e BALEOTTI, Francisco Emílio. A sentença coletiva e a efetividade dos direitos transindividuais. Revista Jurídica Cesumar, Vol. 12, No 2, 2012.

BELLINETTI, Luiz Fernando; SOUZA, Raíssa Fabris. Obstáculos para a efetividade da tutela de direitos transindividuais por entidades sindicais em prejuízo ao efetivo acesso à justiça. Revista Cidadania e Acesso à Justiça, Florianópolis, SC, v. 5, p. 1-17, 2019.

DIDIER JUNIOR, Fredie; ZANETI JUNIOR, Hermes. Curso de Direito Processual Civil: processo coletivo. 9. ed. Salvador: JusPodivm, 2014.

HACHEM, Daniel Wunder. A dupla titularidade (individual e transindividual) dos direitos fundamentais e econômicos, sociais, culturais e ambientais. Revista de Direitos Fundamentais e Democracia, v. 14, n. 14, Curitiba, p. 618-688, jul./dez. 2013.

NEVES, Daniel Amorim Assumpção. Manual de processo coletivo. São Paulo: Método, 2016.

SANTOS, Enoque Ribeiro dos. Processo coletivo do trabalho. 2. ed., rev., atual. e ampl. Rio de Janeiro: Forense, 2018.

ZAVASCKI, Teori Albino. Processo coletivo: tutela de direitos coletivos e tutela coletiva de direitos. Tese (Doutorado Programa de Pós-graduação em Direito) Faculdade de Direito da Universidade Federal do Rio Grande do Sul, Porto Alegre, 2005.

Soumis: Décembre, 2021.

Approbation: Décembre 2021. 